\title{
Case study of intracerebral recurrence of plasmablastic myeloma with achievement of complete response after treatment with lenalidomide
}

\begin{abstract}
We describe an unusual case of multiple myeloma (MM) with plasmablastic morphology in a young man who, after chemotherapy and autologous stem cell transplant, developed disease recurrence with isolated CNS involvement and developed complete remission after treatment with lenalidomide. Plasmablastic type is characterized by immature cells with round nuclei and prominent central nucleoli. According to different studies, plasmablastic MM may account for $15-30 \%$ of MM cases and tend to be associated with adverse prognosis. It has been shown to correlate with high proliferation rate, extensive bone marrow infiltration, but not with high risk chromosomal aberrations. Despite the fact that plasmablastic morphology is not uncommon in MM patients, CNS is involved in less than $1 \%$ of patients with MM. In $82 \%$ of patients with CNS myelomatosis, neoplastic plasma cells were found in CSF; and, in those without CSF involvement, diagnosis was established by MRI which detected either leptomeningeal or intraparenchymal involvement. It is worth mentioning that CNS involvement in MM tends to occur in younger patients, without evidence of advanced disease, sometime with isolated CNS involvement. In many of these patients, complete remission can be achieved with combined treatment which includes systemic chemotherapy, radiation treatment, as well as novel immunomodulating agents. CNS myelomatosis must be suspected whenever patient with MM develops neurologic symptoms not related to chemotherapy treatment.
\end{abstract}

Volume 2 Issue 3 - 2017

\author{
Hoda Elshoni,' Julie Stakiw, ${ }^{2}$ Mark Podberezi' \\ 'Department of Pathology and Laboratory Medicine, University \\ of Saskatchewan, Canada \\ ${ }^{2}$ Department of Hematology \& Oncology, University of \\ Saskatchewan, Canada
}

Correspondence: Mark Podberezin, Department of Pathology and Laboratory Medicine, University of Saskatchewan, 103 Hospital Dr, Saskatoon, SK, S7N OW, Canada,Tel I-306-6552185, Email Mark.Podberezin@saskatoonhealthregion.ca

Received: December 14, 2016 | Published: July 10, 2017

\section{Introduction}

Multiple myeloma (MM) is a disease caused by proliferation of neoplastic clonal plasma cells producing monoclonal immunoglobulin. The disease is manifested by multiple destructive bone lesions, can be associated with anemia, hypercalcemia, and renal failure. The diagnosis is confirmed by bone marrow infiltration with plasma cells and detection of monoclonal protein secretion by serum and urine electrophoresis. Patients usually achieve good clinical response to multi-agent chemotherapy and novel agents such as immunomodulating drug Lenalidomide, immunotherapeutic agents ( e.g. Daratumumab, Elotuzumab, Nivolumab) and proteasome inhibitors (Bortezomib, or Velcade), as well as autologous stem cell transplantation. However, due to persistent minimal residual disease, relapse is eventually imminent. In about one third of MM patients, neoplastic cells demonstrate plasmablastic morphology which is designated as plasmablastic type of $\mathrm{MM} .{ }^{1}$ Involvement of central nervous system (CNS) is observed in less than $1 \%$ of all MM patients ${ }^{2,3}$ and is diagnosed either by presence of neoplastic plasma cells in $\mathrm{CSF}^{4}$ or evidence of intraparenchymal or meningeal involvement as detected by MRI. The largest study was performed in Mayo Clinic and included over 4000 MM patients observed over 16year period. ${ }^{2}$ Symptoms include headache, diplopia, limb weakness, nausea/vomiting, dysphagia, and facial palsy. Patients with MM involving CNS tend to have very short survival despite the use of combined multi-modality treatment. We describe a case of complete regression of intracranial multiple myeloma with plasmablastic morphology when patient achieved complete remission with the use of lenalidomide which is a novel immunomodulatory drug. To the best of our knowledge, this is first published case of intracerebral MM with plasmablastic morphology when complete remission was achieved with the lenalidomide treatment.

\section{Case description}

We describe a case of a 37 year old man who was diagnosed with a stage II IgG Kappa multiple myeloma in 2011 after he had presented with a four-month history of pain in his left hip. Imaging studies showed multiple bone involvement in left proximal femur, lower spine, pelvis and skull. No other physical or imaging finding were appreciated at that time apart from cranial nerve 6 palsy two months prior that was thought to be due to chronic infection. Laboratory data showed $\mathrm{Hb} 66 \mathrm{~g} / \mathrm{L}(\mathrm{N}: 135-180 \mathrm{~g} / \mathrm{L})$, Albumin $30 \mathrm{~g} / \mathrm{L}$ (N:35-52g/L), B2-microglobulin 3.56mg/L (N:0.5-2.2mg/L). Serum protein electrophoresis demonstrated monoclonal band of $44 \mathrm{~g} / \mathrm{L}$ in the elevated Gamma region 47.3g/L (N: 6-12 g/L).

Bone marrow (BM) samples (Figure 1) obtained from left femur and right iliac crest showed the presence of a sheet - like arrangement of plasma cells. Most of plasma cells showed plasmablastic morphology (defined as the presence of immature plasma cells with irregular nucleus, fine vesicular chromatin, and prominent eosinophilic nucleoli). Immunophenotyping showed clonal, kappa light chain restricted neoplastic plasma cells, with partial expression of EMA and CD79a. Cells were positive for CD138 and negative for CD20, Cyclin D1, CD34, Tdt, and CD117. Immunoglobulin heavy chain gene was polymerase chain reaction (PCR) amplified; and amplification product analysis by capillary electrophoresis showed monoclonal IgG kappa. Cytogenetic analysis for $\mathrm{t}(4 ; 14), \mathrm{t}(14 ; 16)$, as well as testing for TP53 and ATM gene mutations revealed no abnormalities. Patient was 
treated with Dexamethasone for four days and subsequently received six cycles of Cyclophosphamide, bortezomib and dexamethasone (CYBOR-D), Later, he underwent autologous stem cell transplant (auto-SCT) and radioimmunotherapy.

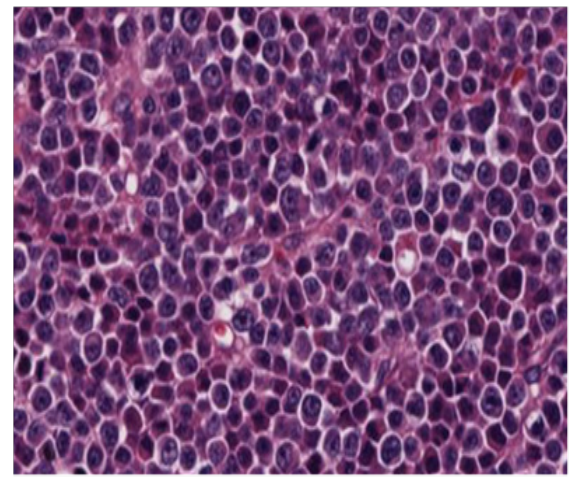

(A)

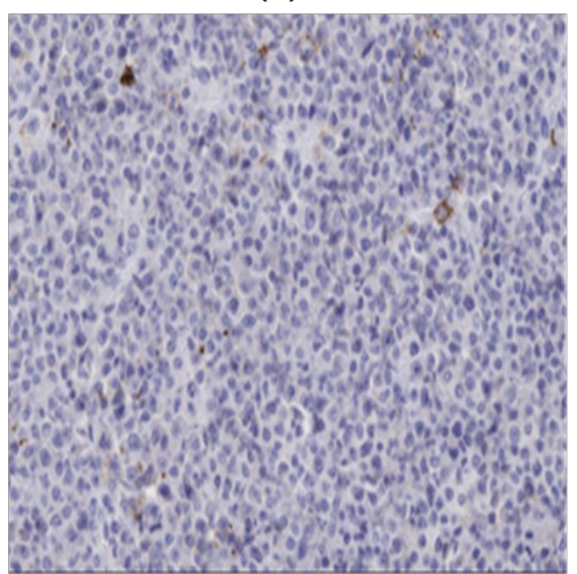

(B)

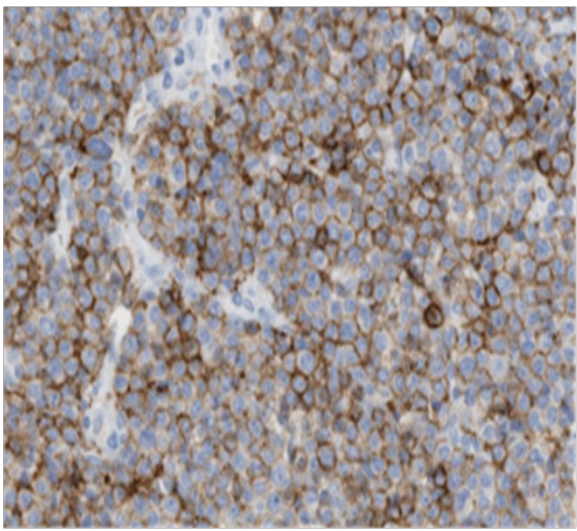

(C)

Figure I Initial bone marrow biopsy in 20I I. (A) Plasma cell myeloma with predominant plasmablastic morphology. (B) \& (C) CD20 negative and CDI38 positive respectively.

After auto-SCT, patient achieved complete remission which lasted for three years. After that, he started experiencing increased headache and confusion. At that time, MRI imaging was performed which showed extensive destructive process involving right clivus and extending into the adjacent petrous temporal bone with no obvious soft tissue involvement. Two months later, right anterior frontal mass
$(1.6 \times 1.57 \mathrm{~cm})$, as well as multiple small foci involving the subcortical white matter of the left frontal lobe and the parietal lobe, were detected. There were no other extramedullary lesions. Laboratory studies showed high creatinine of $111 \mathrm{umol} / \mathrm{L}$, elevated paraprotein $(8 \mathrm{~g} / \mathrm{L})$, with an elevated serum IgG level of $14.2 \mathrm{~g} / \mathrm{L}$.

Patient underwent right frontal craniotomy for an open biopsy of the frontal lobe lesion but declined the bone marrow biopsy. Brain biopsy showed infiltration by neoplastic cells with exclusively plasmablastic morphology (Figure 1A). Immunophenotypically, these cells were positive for CD138, BCL2, MUM1 and negative for CD20, PAX5, CD3, CD10, BCL6, CD56, Cyclin D1, CD30 and EBV (Figure 1B) (Figure 1C). They demonstrated $80 \%$ proliferative index and exhibited clonal kappa light chain restriction. Immunoglobulin heavy chain amplification by PCR detected monoclonal product which was of identical size to that detected in previous femoral bone lesions. Patient was treated with Lenalidomide $25 \mathrm{mg}$ for 21 days and has achieved complete remission. MRI imaging showed complete regression of intracranial parenchymal mass and no new bone lesions. Serum protein electrophoresis showed disappearance of monoclonal protein.

\section{Discussion}

Central nervous system (CNS) involvement in plasma cell myeloma is very rare occurring in less than $1 \%$ of cases, usually at relapses. ${ }^{5}$ The International Myeloma Workshop Consensus Panel identified the most important prognostic markers as cytogenetics, International Staging System (ISS) score, proliferation index (PI), and plasma cell morphology. ${ }^{5}$ A study by Moller et al. ${ }^{1}$ concluded that plasmablastic morphology is significantly associated with a number of adverse prognostic markers such as high ISS score, increased PI and extensive bone marrow infiltration, but it is not associated to high risk chromosomal 14 translocations or deletions. ${ }^{1}$ Moreover, review of 92 cases with central nervous system myelomatosis by Nieuwenhuizen L, et al. revealed that median overall survival from the time of diagnosis of CNS involvement was as low as 2 months. ${ }^{6}$ Midline search of the English literature for CNS involvement by Plasmablastic myeloma presenting with either a clivus mass or intracranial parenchymal lesions revealed 4 cases reported by Fassas et al. ${ }^{7}$ \& Vermeiren P et al. ${ }^{8}$ The largest series were reported by Fassas et al who reported 17 patients with plasmablastic morphology, with one of those presenting with intra-parenchymal masses similar to our patient.

Because of its rarity, there are no established guidelines to treatment of CNS involvement by multiple myeloma. Treatment modalities include intrathecal chemotherapy or combination systemic chemotherapies that cross the blood-brain barrier, with or without cranial irradiation. Systemic chemotherapy agents used with mostly transient responses include a combination of doxorubicin, cyclophosphamide, melphalan, cisplatin, and etoposide, bortezomib, and the immunomodulators thalidomide and lenalidomide. Except for cisplatin and etoposide, most of the other chemotherapeutic regimens used to treat CNS myelomatosis, including bortezomib and the immunomodulators thalidomide and lenalidomide, are not known to cross the blood-brain barrier, or there is insufficient information on their CNS penetration. A recent study by Gozzetti A et al., ${ }^{9}$ demonstrated better outcome in patients who received autotransplant and novel agents over patients treated with older drugs with improved overall survival (25months with the use of novel drugs vs. 8 months when treated with conventional chemotherapeutic agents). Mechanism 
of action of Lenalidomide is not yet fully understood, one theory is the inflammatory reaction and angiogenesis induced by the drug that leads to increased vascular permeability and allows some of these drugs to pass through the blood brain barrier. ${ }^{10}$

\section{Conclusion}

In conclusion, our case study showed potential efficacy of novel immunotherapeutic agents in treating $\mathrm{MM}$ patients with CNS involvement. In addition, with increased longevity of MM patients achieved with use of multi-modality treatment, CNS involvement tends to occur more commonly during the course of disease. Therefore, development of neurological symptoms, which are unrelated to chemotherapy, should warrant proper investigation for possibility of CNS involvement.

\section{Acknowledgements}

None.

\section{Conflict of interest}

The author declares no conflict of interest.

\section{References}

1. Møller HE, Preiss BS, Pedersen P, et al. Clinicopathological features of plasmablastic multiple myeloma: a population-based cohort. APMIS. 2015;123(8):652-658.

2. Jonas Paludo, Utkarsh Painuly, Shaji Kumar, et al. Myelomatous involvement of the central nervous system. Clinical Lymphoma Myeloma and Leukemia. 2016;16(11):644-654.
3. Ren H, Zou Y, Zhao Y, et al. Cerebrospinal fluid cytological diagnosis in multiple myeloma with leptomeningeal involvement. Diagnostic Cytopathol. 2016;45(1):66-68.

4. Jurczyszyn A, Grzasko N, Gozzetti A, et al. Central nervous system involvement by multiple myeloma: a multi-institutional retrospective study of 172 patients in daily clinical practice. American Journal of Hematology. 2016;91(6):575-580.

5. Fassas AB, Muwalla F, Berryman T, et al. Myeloma of the central nervous system: association with high-risk chromosomal abnormalities, plasmablastic morphology and extramedullary manifestations. British Journal of Haematology. 2002;117(1):103-108.

6. Nieuwenhuizen L, Biesma DH. Central nervous system myelomatosis: review of the literature. European Journal of Haematology. 2007;80(1):1-9.

7. Fassas AB, Ward S, Muwalla F, et al. Myeloma of the central nervous system: strong association with unfavorable chromosomal abnormalities and other high-risk disease features. Leukemia \& Lymphoma. 2004;45(2):291-300

8. Vermeiren P, Vantilborgh A, Offner F. Myeloma of the central nervous system: report of a single center case series. Acta Clinica Belgica. 2011;66(3):205-208

9. Gozzetti A, Cerase A, Lotti F, et al. Extramedullary intracranial localization of multiple myeloma and treatment with novel agents: a retrospective survey of 50 patients. Cancer. 2012;118(6):1574-1584.

10. Hattori Y, Iguchi T. Thalidomide for the treatment of multiple myeloma. Congenital Anomalies. 2004;44(3):125-136. 